Chauvelin DouH ${ }^{1,2,3}$

Anaïs-Pasiphaé GoreL ${ }^{1,2}$

Kasso DAïNOU 4,5

Davy FONTEYN ${ }^{2}$

Esther BUSTILLO ${ }^{2}$

LOUISe OBSOMER ${ }^{2}$

AXel BOURDOUXHE ${ }^{2}$

Julie MORIN-RIVAT ${ }^{1,6}$

Éric FORNI ${ }^{7,8}$

SYlvie GOURLET-FLEURY ${ }^{7,8}$

Jean Joël LouMETo ${ }^{3}$

Jean-Louis DouCET ${ }^{1,2}$

${ }^{1}$ TERRA Teaching and Research Center

Forest is life

Gembloux Agro-Bio Tech

Liège Université

Passage des Déportés, 2-5030 Gembloux

Belgium

${ }^{2}$ Université de Liège

Gembloux Agro-Bio Tech

Département BIOSE

Passage des Déportés 2

5030 Gembloux

Belgique

${ }^{3}$ Université Marien Ngouabi

Faculté des sciences et techniques

École Nationale Supérieure d'Agronomie

et de Foresterie

Département des science et technique

forestières

BP 69, Brazzaville

République du Congo

${ }^{4}$ Nature+ asbl / TERRA

Université de Liège

Central African Forests

Gembloux Agro-Bio Tech

Passage des Déportés 2

5030 Gembloux

Belgique

${ }^{5}$ Université Nationale d'Agriculture BP 43, Kétou

Bénin

${ }^{6}$ Musée royal de l'Afrique centrale

Département de biologie,

chimie et géographie

Université du Québec à Rimouski

300 allée des Ursulines

Rimouski QC, G5L 3A1

Canada

${ }^{7}$ Cirad

Forêts et Sociétés

34398 Montpellier

France

${ }^{8}$ Forêts et Sociétés

Univ Montpellier

Cirad

Montpellier

France

Auteur correspondant /

Corresponding author:

Chauvelin Douh - cdouh@doct.ulg.ac.be ;

\title{
Banque de graines du sol et déterminants de la germination du tali, Erythrophleum suaveolens (Guill. \& Perr.) Brenan
}

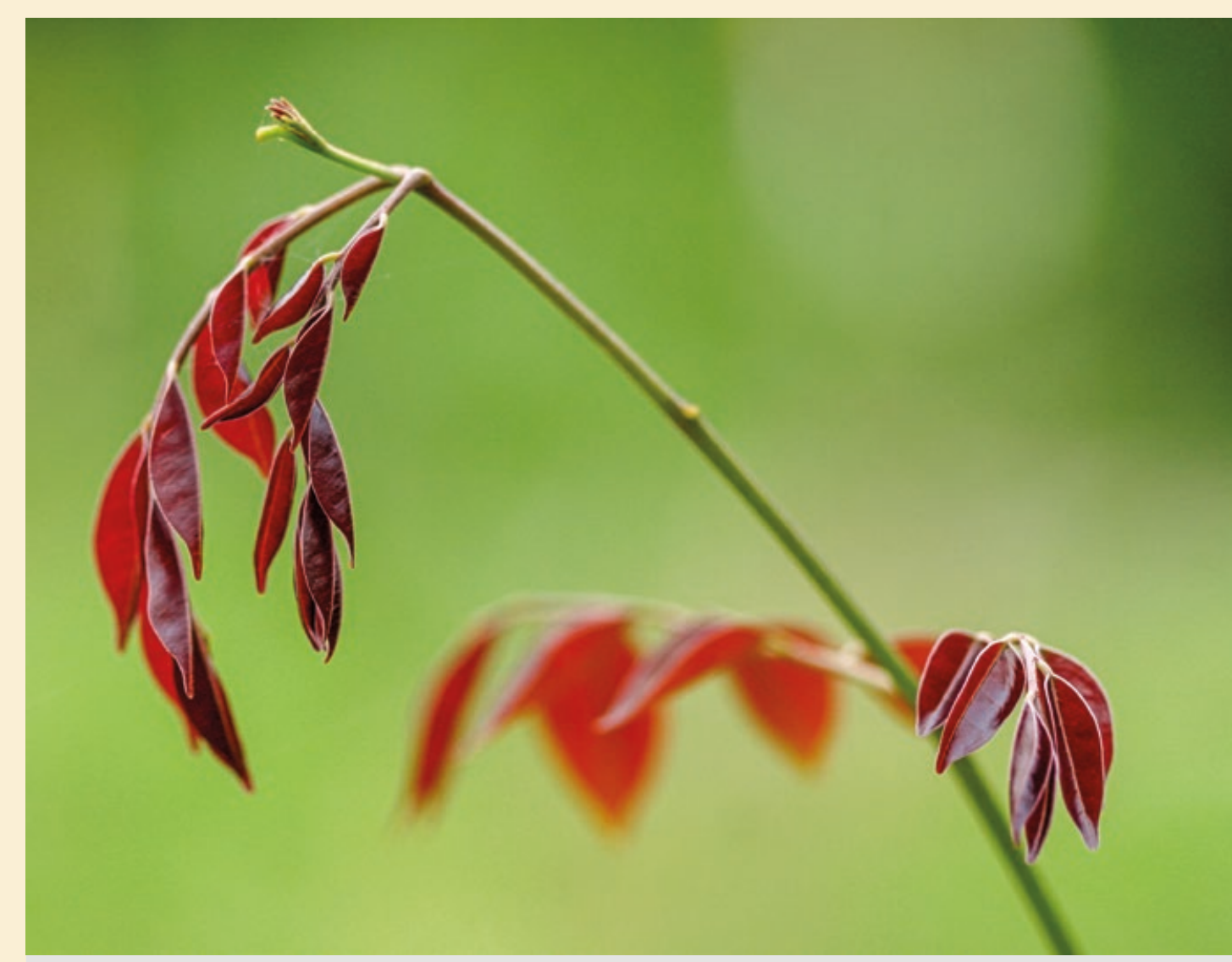

Photo 1.

Feuilles et folioles de l'espèce Erythrophleum suaveolens (tali) en cours de développement. Photo J.-L. Doucet.

Doi : 10.19182/bft2018.338.a31681 - Droit d'auteur (C) 2018, Bois et Forêts des Tropiques @ Cirad - Date de soumission : 25 février 2018 ; date d'acceptation : 25 juin 2018 ; date de publication : $1^{\text {er }}$ décembre 2018.
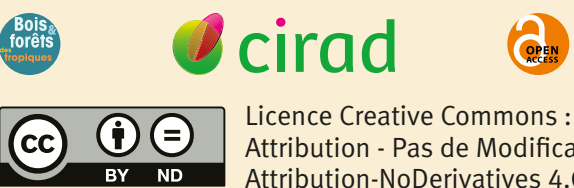

Licence Creative Commons :

Attribution - Pas de Modification 4.0 International.

Attribution-NoDerivatives 4.0 International (CC BY-ND 4.0)
Citer l'article / To cite the article

Douh C., Gorel A.-P., Daïnou K., Fonteyn D., Bustillo E., Obsomer L., Bourdouxhe A., Morin-Rivat J., Forni E., Gourlet-Fleury S., Loumeto J. J., Doucet J.-L., 2018. Banque de graines du sol et déterminants de la germination du tali, Erythrophleum suaveolens (Guill. \& Perr.) Brenan. Bois et Forêts des Tropiques, $n^{\circ} 338$ : 43-55. Doi : https://doi.org/10.19182/bft2018.338. a31681 


\section{RÉSUMÉ}

\section{Banque de graines du sol et déterminants de la germination du tali, Erythrophleum suaveolens (Guill. \& Perr.) Brenan}

Cette étude évalue l'abondance des graines d'Erythrophleum suaveolens dans la banque du sol des forêts denses humides d'Afrique centrale. Les travaux ont été menés au Nord-Congo dans deux types forestiers : la forêt à Celtis sur des sols argilo-sableux à sablo-argileux et la forêt à Manilkara sur des sols sableux. Les tiges d'E. suaveolens $(\mathrm{dhp} \geq 10 \mathrm{~cm}$ ) ont été inventoriées dans deux parcelles de 400 ha, et les structures diamétriques de leurs populations ont été comparées. En outre, 80 fosses ( $2 \times 40$ fosses par type de forêt) ont été creusées aux pieds de 20 arbres (10 par forêt), sur trois couches contiguës de $10 \mathrm{~cm}$ chacune, soit à une profondeur totale de $30 \mathrm{~cm}$, et l'abondance des graines dans la banque du sol a été évaluée. La dormance des graines récoltées a été testée par des essais de germination après traitement au $\mathrm{H}_{2} \mathrm{SO}_{4}$ et cinq graines prélevées jusqu'à une profondeur de $20 \mathrm{~cm}$ dans la forêt à Celtis ont été utilisées pour estimer leur âge par Spectroscopie de Masse par Accélérateur (SMA). La comparaison des structures diamétriques indique une plus grande proportion de tiges de faible diamètre dans la forêt à Celtis. Alors que les densités de tiges $(\mathrm{dhp} \geq 10 \mathrm{~cm})$ sont proches, avec 0,85 et 1,05 tige/ha respectivement, dans la forêt à Celtis et la forêt à Manilkara, les densités de graines sont significativement plus élevées dans la forêt à Celtis $\left(8,55\right.$ graines $\left./ \mathrm{m}^{2}\right)$ que dans la forêt à Manilkara $\left(0,15\right.$ graine $\left./ \mathrm{m}^{2}\right)$. Le pourcentage maximum de germination obtenu était de 19,1\% pour des graines n'ayant subi aucun traitement. Les lots traités à l'acide ont présenté de moindres taux de germination. Ces graines pourraient se conserver une dizaine d'années dans la banque du sol. Les facteurs pouvant influencer les variations de densité des graines sont discutés et des recommandations sylvicoles sont formulées.

Mots-clés : Erythrophleum suaveolens, type de forêt, densité, banque de graines du sol, germination, forêt dense humide, Afrique centrale.

\section{ABSTRACT}

\section{Soil seed bank and determinants of Tali seeding, Erythrophleum suaveolens (Guill. \& Perr.) Brenan}

This study assesses the abundance of Erythrophleum suaveolens seeds in the soil bank of central African moist forests. The work was carried out in two types of forests in northern Congo: the Celtis forest, growing on clay-sandy to sandy-clay soils and the Manilkara forest, growing on sandy soils. E. suaveolens stems $(\mathrm{dbh} \geq 10 \mathrm{~cm})$ were counted in two 400 ha plots, and the diametric structures of the stands were compared. To assess seed abundance in the soil bank, 80 pits ( $2 \times 40$ pits per forest type) were then dug at the foot of 20 trees (10 per forest), removing three contiguous layers of $10 \mathrm{~cm}$ each, i.e. to a total depth of $30 \mathrm{~cm}$. Dormancy of the seeds collected was tested by germination trials after treatment with $\mathrm{H}_{2} \mathrm{SO}_{4}$. Five seeds taken in the Celtis forest down to a depth of $20 \mathrm{~cm}$ were used to estimate their age by Accelerator Mass Spectroscopy (AMS). The comparison of diametric structures indicates a higher proportion of small diameter stems in the Celtis forest. While stem densities $(\mathrm{dbh} \geq 10 \mathrm{~cm})$ were similar in the Celtis and Manilkara forests at 0.85 and 1.05 stems/ha respectively, seed densities were significantly higher in the Celtis forest $\left(8.55\right.$ seeds $\left./ \mathrm{m}^{2}\right)$ than in the Manilkara forest $\left(0.15 \mathrm{seed} / \mathrm{m}^{2}\right)$. The maximum percentage of germination obtained was $19.1 \%$ for seeds having undergone no treatment. Germination rates in the acid-treated batches were lower. These seeds could remain intact in the soil bank for ten years. The factors that could influence seed density variations are discussed and silvicultural recommendations are formulated.

Keywords: Erythrophleum suaveolens, germination, forest type, density, soil seed bank, moist forest, central Africa.

\section{Banco de semillas del suelo y determinantes de la germinación del tali, Erythrophleum suaveolens (Guill. \& Perr.) Brenan}

Este estudio evalúa la abundancia de las semillas de Erythrophleum suaveolens en el banco de semillas del suelo de los bosques densos húmedos de África central. El trabajo se llevó a cabo en el Congo norte en dos tipos forestales: el bosque de Celtis en suelos de arcilloarenosos a arenoarcillosos y el bosque de Manilkara en suelos arenosos. Los tallos de E. suaveolens $(\mathrm{dhp} \geq 10 \mathrm{~cm})$ fueron inventariados en dos parcelas de 400 ha, y se compararon las estructuras diamétricas de sus poblaciones. Además, se cavaron 80 hoyos ( $2 \times 40$ hoyos por tipo de bosque) a los pies de 20 árboles (10 por bosque), en tres capas contiguas de $10 \mathrm{~cm}$ cada una, es decir, a una profundidad total de $30 \mathrm{~cm}$, y se evaluó la abundancia de semillas en el banco de semillas del suelo. Se probó la dormancia de las semillas recogidas mediante ensayos de germinación después de tratarlas con $\mathrm{H}_{2} \mathrm{SO}_{4}$, y se extrajeron cinco semillas hasta una profundidad de $20 \mathrm{~cm}$ en el bosque de Celtis para estimar su edad por espectroscopia de masas con acelerador (AMS). La comparación de las estructuras diametrales indica una mayor proporción de tallos de diámetro pequeño en el bosque de Celtis. Mientras que las densidades de tallos ( $\mathrm{dhp} \geq 10 \mathrm{~cm}$ ) son cercanas, con 0,85 y 1,05 tallos/ha, respectivamente en el bosque de Celtis y el bosque de Manilkara, las densidades de semillas son significativamente más elevadas en el bosque de Celtis ( 8,55 semillas $\left./ \mathrm{m}^{2}\right)$ que en el bosque de Manilkara $\left(0,15\right.$ semillas $\left./ \mathrm{m}^{2}\right)$. El porcentaje máximo de germinación obtenido fue del $19,1 \%$ para semillas que no han sido sometidas a ningún tratamiento. Los lotes tratados con ácido presentaron tasas de germinación menores. Estas semillas parecen poder conservarse un decenio en el banco del suelo. Se tratan los factores que pueden influir en las variaciones de densidad de las semillas y se formulan recomendaciones silvícolas.

Palabras clave: Erythrophleum suaveolens, tipo de bosque, densidad, banco de semillas del suelo, germinación, bosque denso húmedo, África central. 


\section{Introduction}

Les forêts de production d'Afrique centrale font l'objet d'un effort d'aménagement croissant, que l'on estime aujourd'hui à $40 \%$ de la superficie totale des concessions forestières (Bayol et al., 2014). Au cœur du processus figure le plan d'aménagement qui repose notamment sur le calcul des taux de reconstitution des populations d'essences commerciales (Durrieu de Madron et Forni, 1997). Selon les normes des pays concernés, les taux de reconstitution à l'issue d'une rotation doivent atteindre une valeur minimale; à défaut, le diamètre minimum d'exploitation doit être ajusté. Ces taux de reconstitution dépendent de la croissance, de la mortalité et du recrutement des populations (Picard et Gourlet-Fleury, 2008). Le faible taux de recrutement actuel des espèces héliophiles est une contrainte majeure dans le processus d'aménagement et la gestion sur le long terme de leurs populations semble compromise en l'absence d'interventions sylvicoles mûrement réfléchies (Karsenty et Gourlet-Fleury, 2006). La régénération des espèces héliophiles ne peut se faire que dans les milieux ouverts naturellement (chablis) ou artificiellement (champs, trouées d'abattage, routes abandonnées). Trois grands ensembles participent à la cicatrisation de tels milieux : le potentiel végétatif, le potentiel séminal advectif et le potentiel séminal édaphique (Lescure et al., 1989). Le potentiel végétatif représente l'ensemble des individus ayant survécu à la perturbation. Le potentiel séminal advectif correspond aux apports postérieurs à l'ouverture du couvert forestier.

Enfin, le potentiel séminal édaphique, ou banque de graines du sol, est défini comme un ensemble de graines viables non germées enfouies dans le sol. La banque de graines du sol est donc une composante importante de la dynamique des populations (Warr et al., 1993), mais demeure très peu étudiée en Afrique centrale (Douh et al., 2014).

Les rares études effectuées en la matière démontrent pourtant une forte densité de graines viables dans le sol, au moins jusqu'à $20 \mathrm{~cm}$ de profondeur, les horizons plus profonds n'ayant pas encore été étudiés (Douh et al., 2018).

Les graines se répartissent en deux groupes. Le premier est constitué des graines récalcitrantes qui perdent rapidement leur viabilité lorsqu'elles sont stockées, en raison d'une teneur en eau élevée. Le second comprend les graines orthodoxes, qui peuvent intégrer la banque de graines du sol et y survivre plusieurs années (Swaine et Whitmore, 1998 ; Baskin et Baskin, 2003). Ces dernières graines sont généralement en dormance, laquelle peut être définie comme le processus physiologique ou mécanique empêchant leur germination immédiate (Baskin et Baskin, 2003). Ce mécanisme concerne un nombre réduit d'espèces productrices de bois d'œuvre en Afrique centrale. L'espèce Erythrophleum suaveolens (Guill. \& Perr.) Brenan (Daïnou et al., 2011) en fait partie.

Erythrophleum suaveolens est une espèce commerciale des forêts denses humides d'Afrique tropicale. Cet arbre de la famille des Leguminoseae-Detarioideae, connu sous le nom commercial de "tali » (nomenclature ATIBT), est exploité pour la qualité de son bois très prisé, notamment en menuiserie et pour les travaux lourds (traverses de chemin de fer, ouvrages de ports, quais et ponts) (Okeyo, 2006). Selon l'Organisation internationale des bois tropicaux (OIBT), E. suaveolens fait partie des essences les plus exploitées du Bassin du Congo. Le Cameroun et le Congo en sont les principaux exportateurs (OIBT, 2012 ; de Wasseige et al., 2014). L'espèce présente une large distribution en Afrique (Aubréville, 1970 ; Hawthorne, 1995 ; Duminil et al., 2010 ; Gorel et al., 2015).

La dispersion primaire des graines est ballochore (Hawthorne, 1995 ; Doucet, 2003), et une dispersion secondaire, assurée par les rongeurs, serait possible (Guion, 2011). Plus rarement, elle serait exercée par certains primates qui seraient attirés par le mucilage entourant les graines (Koné et Lambert, 2008 ; Petre et al., 2013). Les graines sont orthodoxes et la dormance des graines collectées sous les semenciers peut être levée par un traitement à l'acide sulfurique à $95 \%$ pendant 40 minutes (Delhaye, 2006).

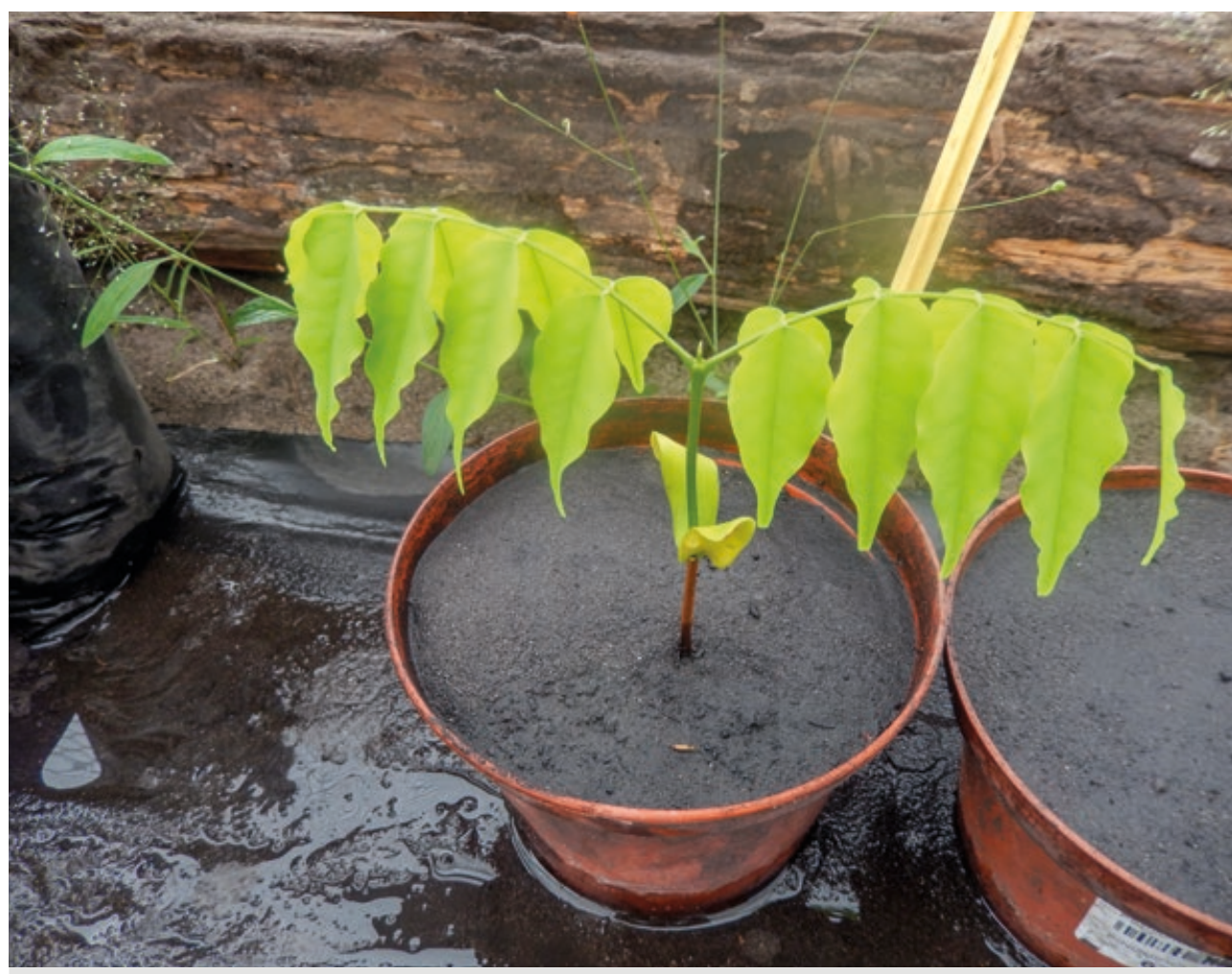

Photo 2.

Plantule de l'espèce Erythrophleum suaveolens (tali). Photo C. Douh. 
Dotée d'un tempérament héliophile (Hawthorne, 1995), Erythrophleum suaveolens souffre généralement d'un déficit de régénération, lequel se traduit, en forêt mature, par une distribution gaussienne des effectifs par classe diamétrique (Poorter et al., 1996 ; Durrieu de Madron et Forni, 1997).

L'abondance actuelle de l'espèce dans les forêts d'Afrique centrale serait liée à une forte régénération dans des champs installés vers la fin du XIXe siècle par les agriculteurs itinérants (Morin-Rivat et al., 2017).

Dans un tel contexte, l'exploitation forestière pourrait soit accélérer la régression de l'espèce en prélevant les semenciers, soit offrir des conditions propices à la germination des graines présentes dans le sol. Pour trancher la question et garantir une gestion des populations de cette espèce sur le long terme, il convient de quantifier l'abondance des graines stockées dans le sol sous différents types forestiers et de mieux comprendre les mécanismes impliqués dans la levée de la dormance.

Nos hypothèses sont les suivantes : les densités d'adultes étant similaires, les densités de graines d'Erythrophleum suaveolens présentes dans la banque du sol devraient l'être aussi, et ne sont pas influencées par le type de forêt ; les densités de graines d'E. suaveolens varient en fonction de la profondeur dans le sol, et peuvent être présentes à plus de $20 \mathrm{~cm}$; les graines d'E. suaveolens enfouies dans la banque du sol peuvent en conséquence se conserver durant plusieurs années.

\section{Matériel et méthodes}

\section{Sites d'étude}

L'étude a été menée dans deux types de forêts d'Afrique centrale, au sein des dispositifs du projet DynAfFor (Dynamique des forêts d'Afrique centrale ${ }^{1}$ ), au Nord de la République du Congo.

Le premier site, dans la concession forestière attribuée à CIB/OLAM, proche de la localité de Loundoungou, se trouve dans une forêt à Celtis, tandis que le second site, au sein de la concession forestière de Mokabi-Dzanga, est localisé dans une forêt à Manilkara. Les deux types de forêts ${ }^{1}$ http://www.dynaffor.org diffèrent par leur composition floristique et leur substrat pédologique : la forêt à Celtis est semi-décidue, reposant sur des sols argilo-sableux à sablo-argileux typiques des alluvions, tandis que la forêt à Manilkara est une forêt comprenant davantage d'espèces sempervirentes, reposant sur des sols sableux à argilo-sableux typiques des grès de Carnot (Gond et al., 2013 ; Fayolle et al., 2014 ; Freycon, 2014) (tableau I). En termes de fertilité chimique, les sols de la forêt à Manilkara sont supposés plus pauvres que ceux de la forêt à Celtis. La forêt à Celtis a été régulièrement perturbée par le passé (Oslisly et al., 2013 ; Morin-Rivat et al., 2014) par des activités humaines traditionnelles et peut être qualifiée de vieille forêt secondaire (White, 1983). La forêt à Manilkara, est plus ancienne et moins perturbée que la forêt à Celtis (Oslisly et al., 2013 ; Morin-Rivat et al., 2014).

\section{Structures des populations d'Erythrophleum suaveolens}

Tous les semenciers d'E. suaveolens de diamètre à hauteur de poitrine $(\mathrm{dhp}) \geq 10 \mathrm{~cm}$ ont été inventoriés et cartographiés dans deux parcelles de 400 ha. Cet inventaire a permis de caractériser les structures des populations de l'espèce (distribution des effectifs par classe de diamètre).

\section{Dispositif expérimental et tests de germination}

En 2014, dans chaque parcelle de 400 ha, un semencier d'E. suaveolens a été choisi aléatoirement par classe de diamètre, dans une gamme de dhp variant de 30 à $120 \mathrm{~cm}$ (répartis en dix classes de diamètre), procurant un total de 10 semenciers par site. Le diamètre de $30 \mathrm{~cm}$ correspond au diamètre minimal de reproduction de l'espèce (Doucet, 2003 ; Kouadio, 2009). Quatre fosses de surface unitaire de $1 \mathrm{~m} \times 1 \mathrm{~m}$ ont été creusées à une distance de $5 \mathrm{~m}$ de la base de chaque semencier dans les quatre directions cardinales (annexe 1). Au total, 80 fosses ont donc été creusées pour l'ensemble des deux sites. Le sol a été prélevé minutieusement sur trois couches contiguës de $10 \mathrm{~cm}$ chacune (continuellement vérifiées à l'aide d'un étalon de $10 \mathrm{~cm}$ de long) à l'aide d'une bêche et d'une truelle, sur une profondeur totale de 0 à $30 \mathrm{~cm}$ (annexe 1). L'échantillon de sol prélevé par couche a été tamisé (mailles de $2 \mathrm{~mm}$ de largeur) et les graines d'E. suaveolens trouvées ont été collectées.

\section{Tableau I.}

Description synthétique des deux types de forêts (forêt à Celtis et forêt à Manilkara) (Verelst, 2009 ; Fayolle et al., 2012 ; Freycon, 2014), en tenant compte des précipitations relevées entre 2000 et 2008 à Ouesso, Impfondo et Mbaïki.

\begin{tabular}{|c|c|c|}
\hline & Forêt à Celtis (F-Celt) & Forêt à Manilkara (F-Mani) \\
\hline Coordonnées géographiques & $\begin{array}{l}02^{\circ} 18^{\prime}-02^{\circ} 22 \mathrm{~N} \\
17^{\circ} 31^{\prime}-17^{\circ} 34^{\prime} \mathrm{E}\end{array}$ & $\begin{array}{l}03^{\circ} 01^{\prime}-03^{\circ} 37^{\prime} \mathrm{N} \\
16^{\circ} 30^{\prime}-17^{\circ} 22^{\prime} \mathrm{E}\end{array}$ \\
\hline Altitude (m) & $410-460$ & $510-570$ \\
\hline Pluviométrie annuelle (mm/an) & 1729 & 1670 \\
\hline Type de sol & Acrisol-Arenosol-Gleysol & Acrisol-Arenosol \\
\hline Géomorphologie & Piedmont au bord de la cuvette congolaise & Plateau à grès de Carnot \\
\hline Type de forêt & Semi-sempervirent & Semi-sempervirent et sempervirent \\
\hline
\end{tabular}


Afin d'estimer l'âge des graines dans la banque du sol, cinq graines ont été prélevées dans deux couches différentes au sein de la forêt à Celtis : quatre graines dans la couche 0-10 cm, et une graine dans la couche $10-20 \mathrm{~cm}$. La forêt à Celtis et les couches susmentionnées ont été retenues pour la datation car étant les plus représentatives de la majorité des graines récoltées, contrairement à la forêt à Manilkara où la banque de graines du sol d'E. suaveolens s'est avérée quasi inexistante (voir le chapitre des résultats).

La datation des cinq graines a été effectuée par la méthode radiocarbone utilisant la Spectroscopie de Masse par Accélérateur (SMA) au London BioScience Innovation Centre (Beta Analytics). La méthode de datation via la SMA permet d'obtenir des comptages isotopiques très précis sur des échantillons individuels en n'utilisant que de très petites quantités de carbone ( $100 \mu \mathrm{g})$ (Donahue, 1995 ; Moriuchi et al., 2000), ce qui était adapté pour la datation des graines.

La calibration des datations obtenues a été effectuée en utilisant le programme OxCal (Bronk Ramsey, 1995 ; Hua et al., 2013 ; Reimer et al., 2013). Les graines restantes ont été conservées pendant 17 mois dans des enveloppes avant les tests de germination. Compte tenu du nombre très faible de graines récoltées dans la forêt à Manilkara et à plus de $10 \mathrm{~cm}$ de profondeur dans la forêt à Celtis (voir résultats), les tests de germination n'ont été effectués qu'à l'aide des semences collectées dans la couche $0-10 \mathrm{~cm}$ de la forêt à Celtis.

Le lot de graines utilisé provient de cinq individus d'E. suaveolens de diamètres compris entre 30 et $70 \mathrm{~cm}$ (tableau II). Pour chaque individu, le lot de graines a été divisé en trois traitements pour tester les modalités suivantes de levée de dormance, à raison de 46 à 47 graines par traitement :

- Trait. 0 = aucun traitement ;

- Trait. 1 = graines trempées dans une solution d'acide sulfurique $\left(\mathrm{H}_{2} \mathrm{SO}_{4}\right)$ diluée à $95 \%$ pendant 20 minutes ;

- Trait. 2 : graines trempées dans une solution d'acide sulfurique $\left(\mathrm{H}_{2} \mathrm{SO}_{4}\right)$ diluée à $95 \%$ pendant 60 minutes.

\section{Tableau II.}

Nombre de graines pour chaque individu et modalités de levée de dormance. Trait.0 : aucun traitement ; Trait. 1 : $\mathrm{H}_{2} \mathrm{SO}$ à $95 \%$ pendant 20 minutes ; Trait. $2 \mathrm{:}_{2} \mathrm{SO}_{4}$ à $95 \%$ pendant 60 minutes.

\begin{tabular}{|c|c|c|c|c|}
\hline \multirow[t]{2}{*}{ Arbres } & \multicolumn{3}{|c|}{ Nombre de graines } & \multirow[b]{2}{*}{ Total } \\
\hline & Trait.0 & Trait.1 & Trait.2 & \\
\hline Tali $n^{\circ} 1$ & 10 & 9 & 9 & 28 \\
\hline Tali $n^{\circ} 2$ & 10 & 10 & 10 & 30 \\
\hline Tali $n^{\circ} 3$ & 10 & 10 & 10 & 30 \\
\hline Tali $n^{\circ} 4$ & 9 & 9 & 9 & 27 \\
\hline Tali $n^{\circ} 5$ & 8 & 8 & 8 & 24 \\
\hline Total & 47 & 46 & 46 & 139 \\
\hline
\end{tabular}

Le choix de ces différentes modalités repose sur des études précédentes (Delhaye, 2006 ; Kouadio, 2009). Après chaque immersion dans l'acide sulfurique, les graines ont été rincées dans de l'eau distillée pendant une heure, en changeant l'eau de rinçage toutes les 20 minutes (Footitt et Cohn, 1995 ; Delhaye, 2006 ; Kouadio, 2009).

Les tests de germination ont été menés en serre à Gembloux Agro-Bio Tech (Université de Liège, Belgique), à une température moyenne de $25^{\circ} \mathrm{C}$ et une humidité moyenne de $60 \%$.

Les graines ont été immédiatement semées après les traitements, mises en terre et recouvertes d'une pellicule de terreau de $3 \mathrm{~mm}$ d'épaisseur le 13 novembre 2016, dans des pots individuels de $0,5 \mathrm{I}$ remplis de deux tiers de terreau et d'un mélange d'un tiers de sol rouge ferralitique récolté sur le terrain. Cette terre ferralitique a été incorporée au substrat pour assurer l'effet du cortège bactérien naturel du sol sur la germination des graines de cette espèce (Diabate et al., 2005). Les graines semées ont été arrosées par un système automatique et la germination a été suivie quotidiennement. L'expérience a pris fin le 2 mars 2017, soit après 16 semaines de suivi. À la fin de l'expérimentation, le nombre de graines ayant effectivement germé, le nombre de graines non germées d'apparence saine et le nombre de graines altérées (infestées, pourries) ont été estimés pour chaque traitement (Danthu, 1993 ; Elazazi, 2016). Le pourcentage de germination a ensuite été calculé (Maguire, 1962). Les graines d'apparence saine ont ensuite été soumises à un « test de coupe de graines » (Ensconet, 2009).

\section{Analyse des données}

Les différences de densités de graines entre les deux types de forêts ont été évaluées à l'aide d'un test non paramétrique de Kruskal-Wallis. Un modèle linéaire généralisé (GLM) binomial à un facteur fixe a été utilisé pour tester l'effet des trois modalités de traitement (McCullagh et Nelder, 1989 ; Hardin et Hilbe, 2007).

\section{Résultats}

\section{Structures des populations et densités de la banque de graines du sol d'E. suaveolens}

Les densités observées sont de 0,85 tige/ha (dhp $\geq$ $10 \mathrm{~cm}$ ) dans la forêt à Celtis et de 1,08 tige/ha dans la forêt à Manilkara. Le nombre d'arbres potentiellement fertiles $(\mathrm{dhp} \geq 30 \mathrm{~cm}$ ) est respectivement de 0,62 et de 0,87 arbre/ha.

La comparaison des structures de population indique une plus forte proportion de jeunes tiges dans la forêt à Celtis (figure 1). Le nombre total de graines collectées dans le sol est de 342 graines pour la forêt à Celtis et de six graines pour la forêt à Manilkara, avec des densités moyennes respectives de 8,55 et 0,15 graine $/ \mathrm{m}^{2}$ (tableau III). La différence de densité de la banque de graines du sol entre les deux types de forêts est significative (test de Kruskal-Wallis ; $\mathrm{H}=7,5 ; p<0,001)$. La densité de graines de l'espèce est surtout concentrée dans la première couche sondée, et aucune graine n'a été observée dans la troisième couche. 


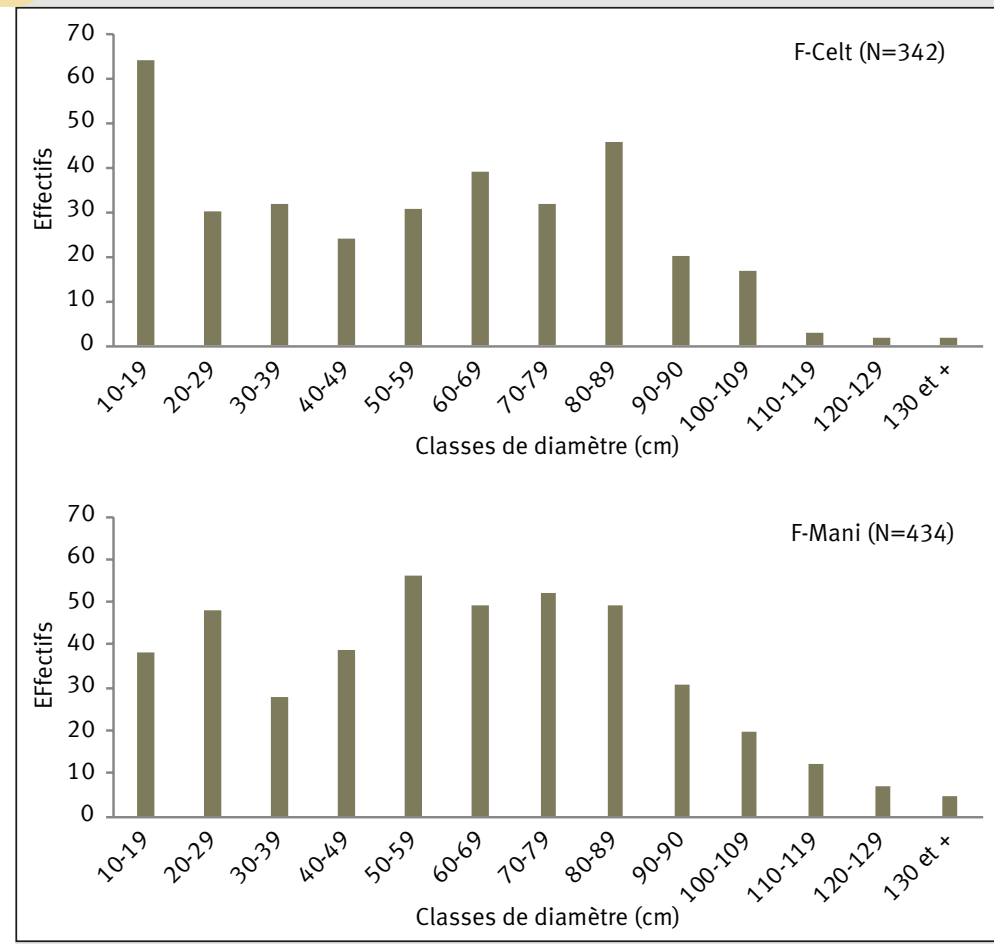

Figure 1.

Structures des populations d'Erythrophleum suaveolens au sein des deux types de forêts, forêt à Celtis (F-Celt) et forêt à Manilkara (F-Mani).

\section{Influence des traitements sur le taux de germination}

Au terme de l'expérience, tous traitements confondus, le pourcentage de germination observé a été faible, puisqu'il n'a atteint que $12,9 \%$ des graines semées. Les graines restantes étaient à $46,8 \%$ des graines non germées d'apparence saine, et à 40,3\% altérées par la pourriture. Plus précisément, les taux de germination étaient respectivement de 19,1\%, 4,3\% et 15,2\% pour des traitements d'intensité croissante (Trait.0, Trait.1 et Trait.2) (figure 2). D’après le GLM, la probabilité de germination des graines est significativement plus faible pour le traitement 1 (tableau IV). La probabilité que les graines demeurent d'apparence saine est significativement plus faible pour le traitement 2. Enfin, c'est également ce dernier traitement qui donne la probabilité d'altération des graines la plus élevée (56\%).

\section{Datation radiocarbone par Spectrométrie de Masse par Accélérateur (SMA)}

Considérant l'année de collecte des graines (2014), les datations indiquent un âge compris entre cinq et neuf ans (annexe 2). En effet, les graines auraient été dispersées avec une forte probabilité au cours des périodes suivantes : 2005-2009 (91,5\%), 2006-2009 (88,5\%), 2007-2009 (68,2 \%), 2007-2009 (80,5\%) et 2007-2009 (85,3\%), respectivement pour les graines F-Celt 01, F-Celt 02, F-Celt 03, F-Celt 04 et F-Celt 05.

\section{Tableau III.}

Nombre de graines collectées dans les deux types de forêts (forêt à Celtis et forêt à Manilkara) par classe de diamètre et par couche de sol. Les lots de graines utilisés pour les tests de germination proviennent des lots indiqués d’un astérisque.

\begin{tabular}{|c|c|c|c|c|c|c|c|c|c|c|c|}
\hline $\begin{array}{l}\text { Couches } \\
\text { de sol }(\mathrm{cm})\end{array}$ & $\begin{array}{c}\varnothing 30-40 \\
(\mathrm{~cm}) \\
\text { Tali } \mathrm{n}^{\circ} 1\end{array}$ & $\begin{array}{c}\varnothing 40-50 \\
(\mathrm{~cm}) \\
\text { Tali } \mathrm{n}^{\circ} 2\end{array}$ & $\begin{array}{c}\varnothing 50-60 \\
(\mathrm{~cm}) \\
\text { Tali } \mathrm{n}^{\circ} 3\end{array}$ & $\begin{array}{c}\varnothing 60-70 \\
(\mathrm{~cm}) \\
\text { Tali } \mathrm{n}^{\circ} 4\end{array}$ & $\begin{array}{c}\emptyset 70-80 \\
(\mathrm{~cm}) \\
\text { Tali } \mathrm{n}^{\circ} 5\end{array}$ & $\begin{array}{c}\varnothing 80-90 \\
(\mathrm{~cm}) \\
\text { Tali n } 6\end{array}$ & $\begin{array}{c}\varnothing 90-100 \\
(\mathrm{~cm}) \\
\text { Tali } \mathrm{n}^{\circ} 7\end{array}$ & $\begin{array}{c}\varnothing 100-110 \\
(\mathrm{~cm}) \\
\text { Tali } \mathrm{n}^{\circ} 8\end{array}$ & $\begin{array}{c}\varnothing 110-120 \\
(\mathrm{~cm}) \\
\text { Tali } \mathrm{n}^{\circ} 9\end{array}$ & $\begin{array}{c}\emptyset 120-130 \\
(\mathrm{~cm}) \\
\text { Tali } \mathrm{n}^{\circ} 10\end{array}$ & $\begin{array}{l}\text { Total de } \\
\text { graines }\end{array}$ \\
\hline \multicolumn{12}{|c|}{ Forêt à Celtis (vieille forêt secondaire) } \\
\hline $0-10$ & $30 *$ & $115^{*}$ & $84^{*}$ & $31^{\star}$ & $26^{*}$ & 14 & 19 & 3 & 8 & 6 & 336 \\
\hline $10-20$ & 0 & 3 & 0 & 3 & 0 & 0 & 0 & 0 & 0 & 0 & 6 \\
\hline $20-30$ & 0 & 0 & 0 & 0 & 0 & 0 & 0 & 0 & 0 & 0 & 0 \\
\hline Total & 30 & 118 & 84 & 34 & 26 & 14 & 19 & 3 & 8 & 6 & 342 \\
\hline \multicolumn{12}{|c|}{ Forêt à Manilkara (forêt ancienne) } \\
\hline $0-10$ & 0 & 0 & 0 & 0 & 0 & 0 & 1 & 0 & 2 & 0 & 3 \\
\hline $10-20$ & 0 & 2 & 0 & 0 & 0 & 0 & 0 & 0 & 1 & 0 & 3 \\
\hline $20-30$ & 0 & 0 & 0 & 0 & 0 & 0 & 0 & 0 & 0 & 0 & 0 \\
\hline Total & 0 & 2 & 0 & 0 & 0 & 0 & 1 & 0 & 3 & 0 & 6 \\
\hline
\end{tabular}




\section{Discussion}

\section{Variation de la banque de graines du sol d'E. suaveolens en fonction des types de forêts}

La densité de graines d'E. suaveolens est significativement plus faible dans la forêt à Manilkara que dans la forêt à Celtis. Une telle différence pourrait résulter de plusieurs facteurs mutuellement non exclusifs : le degré de maturation de la forêt, le climat, l'abondance des semenciers, les communautés de prédateurs et pathogènes de graines.

Toutes espèces confondues, les densités de graines dans les sols forestiers tropicaux varient de 25 à 3350 graines $/ \mathrm{m}^{2}$ (Garwood, 1989). La densité de la banque de graines du sol en forêts matures est inférieure à celle des forêts secondaires (Hall et Swaine, 1980 ; Uhl et al., 1981). Cette variabilité est en partie liée à la composition spécifique des types forestiers au cours de la succession végétale. En effet, les espèces pionnières et/ou héliophiles des forêts perturbées produisent un stock plus important de graines et, par conséquent, présenteraient davantage de graines dormantes dans la banque du sol (Hopkins et Graham, 1983 ; Uhl et Clark, 1983 ; Saulei, 1984 ; Lawton et Putz, 1988) (tableau III). Warr et al. (1993) confirment que les densités de graines diminuent progressivement au gré de la succession végétale. Nos deux types forestiers répondent à la même logique.

Douh et al. (2018) ont mis en évidence des banques de graines, toutes espèces confondues, plus abondantes dans la forêt à Celtis que dans la forêt à Manilkara.

La forêt à Manilkara serait plus âgée que la forêt à Celtis (Fayolle et al., 2014 ; Douh et al., 2018). En effet, Morin-Rivat et al. (2016) ont observé dans les sols de la forêt à Manilkara une moindre abondance de charbon de bois et de tessons de poterie, lesquels sont des indicateurs d'activités anthropiques anciennes.

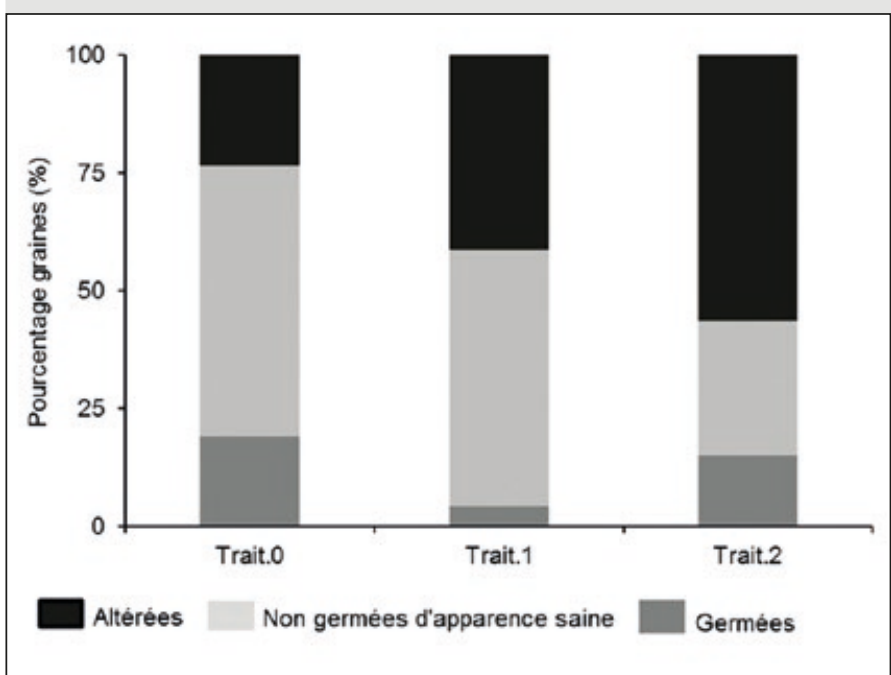

Figure 2.

Pourcentage des graines germées (gris), des graines non germées d'apparence saine (gris clair) et des graines altérées (noir) en fonction des différents traitements. Trait.0 = aucun traitement, Trait. $1=\mathrm{H}_{2} \mathrm{SO}_{4}$ à $95 \%$ pendant 20 minutes et Trait. $2=\mathrm{H}_{2} \mathrm{SO}_{4}$ à $95 \%$ pendant 60 minutes.

Toutefois, le nombre quasi nul de graines dans la banque du sol de la forêt à Manilkara n'est pas lié à une moindre abondance d'arbres adultes. En effet, les densités de pieds potentiellement fertiles $(\mathrm{dhp} \geq 30 \mathrm{~cm})$ sont plus élevées dans cette forêt que dans la forêt à Celtis.

Cependant, certaines espèces pionnières et/ou héliophiles ne fructifient plus quand le stade forestier est trop évolué, notamment lorsqu'elles se retrouvent en dessous de la canopée (Mbarga et al., 1999). Cela pourrait être le cas d'E. suaveolens, car l'espèce atteint tout au plus $40 \mathrm{~m}$ de haut et est donc susceptible d'être surcimée par d'autres espèces dans les forêts très âgées. Ouédraogo et al. (2016)

Tableau IV.

Prédictions et paramètres des modèles linéaires généralisés utilisés, les variables expliquées (probabilités des graines germées, non germées d'apparence saine et altérées) suivant une loi binomiale. ES = erreur standard.

\begin{tabular}{|c|c|c|c|c|c|c|}
\hline & $\begin{array}{l}\text { Paramètres } \\
\text { modèle linéaire }\end{array}$ & $\begin{array}{c}\text { Traitement } 0 \\
\text { (intercept) }\end{array}$ & Traitement 1 & Traitement 2 & Modèle & $\begin{array}{l}\text { AIC } \\
\text { Modèle NULL }\end{array}$ \\
\hline Germées & $\begin{array}{l}\text { Probabilité prédite et ES } \\
\text { Estimation } \\
\text { Erreur standard } \\
\text { Z-value } \\
\text { P-value }\end{array}$ & $\begin{array}{c}19 \pm 6 \% \\
-1,44 \\
0,37 \\
-3,88 \\
<0,001\end{array}$ & $\begin{array}{l}4 \pm 3 \% \\
-1,65 \\
0,81 \\
-2,03 \\
0,04\end{array}$ & $\begin{array}{c}15 \pm 5 \% \\
-0,28 \\
0,55 \\
-0,50 \\
0,61\end{array}$ & 101,59 & 107.15 \\
\hline $\begin{array}{l}\text { Non germées } \\
\text { d'apparence saine }\end{array}$ & $\begin{array}{l}\text { Probabilité prédite et ES } \\
\text { Estimation } \\
\text { Erreur standard } \\
\text { Z-value } \\
\text { P-value }\end{array}$ & $\begin{array}{c}57 \pm 7 \% \\
0,30 \\
0,29 \\
1,02 \\
0,30\end{array}$ & $\begin{array}{c}54 \pm 7 \% \\
-0,12 \\
0,41 \\
-0,30 \\
0,76\end{array}$ & $\begin{array}{c}28 \pm 6 \% \\
-1,23 \\
0,44 \\
-2,80 \\
0,005\end{array}$ & 182.31 & 192.31 \\
\hline Altérées & $\begin{array}{l}\text { Probabilité prédite et ES } \\
\text { Estimation } \\
\text { Erreur standard } \\
\text { Z-value } \\
\text { P-value }\end{array}$ & $\begin{array}{c}23 \pm 6 \% \\
-1,18 \\
0,34 \\
-3,44 \\
0,001\end{array}$ & $\begin{array}{c}41 \pm 7 \% \\
0,83 \\
0,46 \\
1,82 \\
0,07\end{array}$ & $\begin{array}{c}56 \pm 7 \% \\
1,44 \\
0,45 \\
3,18 \\
0,005\end{array}$ & 176.50 & 187.42 \\
\hline
\end{tabular}


confirment que les diamètres de floraison et de fructification d'E. suaveolens sont influencés par le type de forêt et que la probabilité de fructification de l'espèce dépend de l'exposition de sa couronne à la lumière. Par ailleurs, selon les mêmes auteurs, la proportion d'E. suaveolens émergents varie fortement entre les sites.

Les facteurs climatiques (température, pluviométrie, humidité, etc.) peuvent aussi influencer le cycle de floraison et de fructification des espèces d'arbres (Hecketsweiler, 1992 ; Mapongmetsem et al., 1998). Toutefois les climats varient peu entre les deux sites. Selon la classification de Köppen, la forêt à Celtis est soumise au climat de type Am, tandis que la forêt à Manilkara est de type Aw, tout en étant à proximité de la limite avec le climat de type Am (Verelst, 2009; Freycon, 2014).

Un autre facteur explicatif serait une différence dans les communautés animales intervenant dans la prédation et/ou la dispersion secondaire des graines.

En forêt tropicale d'Afrique, Effiom et al. (2013) ont démontré que les rongeurs pourraient proliférer en cas de chasse intense de grands mammifères. Or, l'abondance des rongeurs peut se traduire par une plus grande prédation des graines (Evrard et al., 2017). Il existe bel et bien une importante différence entre les deux types de forêts. La zone étudiée dans la forêt à Manilkara est soumise à une plus forte pression de chasse (Stokes et al., 2010 ; Haurez et al., 2013 ; Mbete et al., 2014) et une possible prolifération des rongeurs.

Guion (2011) ayant confirmé que les graines d'E. suaveolens étaient recherchées par un rongeur (Cricetomys emini), une prédation importante des graines n'est donc pas à exclure. II n'est toutefois pas impossible que ce rongeur puisse aussi participer à une dispersion secondaire des graines via la pratique du scatter-hoarding (Tosso et al., 2017).
Les conditions édaphiques pourraient également jouer un rôle. D'une part, la fertilité du sol pourrait influencer la reproduction des arbres (Cardoso et al., 2012). Sollins (1998) estime que la production de fruits diminue avec la diminution du pH du sol (Proctor et al., 1983). Par conséquent, la production de graines pourrait différer entre les deux sites, compte tenu des caractéristiques différentes des sols. D'autre part, l'acidité et/ou la porosité du sol pourrait influencer la conservation des graines (Chen et al., 2013). Cette dernière hypothèse semble toutefois peu vraisemblable dans notre cas car d'autres graines dormantes et a priori moins résistantes que celles d'E. suaveolens (par exemple, Nauclea diderrichii) ont été observées dans la banque du sol de la forêt à Manilkara (Douh et al., 2018).

Enfin, Augspurger (1983) et Augspurger et Kelly (1984) estiment que les habitats à canopée fermée et ombragée pourraient constituer des zones d'intense activité fongique, entraînant la mortalité des graines dans la banque du sol. Christensen (1989) rappelle à cet effet que les activités fongiques peuvent avoir des effets saprophytes ou pathogènes sur la banque de graines, causant la mortalité des graines.

Par exemple, certains champignons sont des saprophytes généralistes qui se nourrissent des graines viables avec des cellulases et d'autres enzymes dégradantes (Mills, 1983 ; Crist et Friese, 1993). Dalling et al. (1998) démontrent également que la réduction des populations fongiques augmenterait significativement la survie des graines dans la banque du sol.

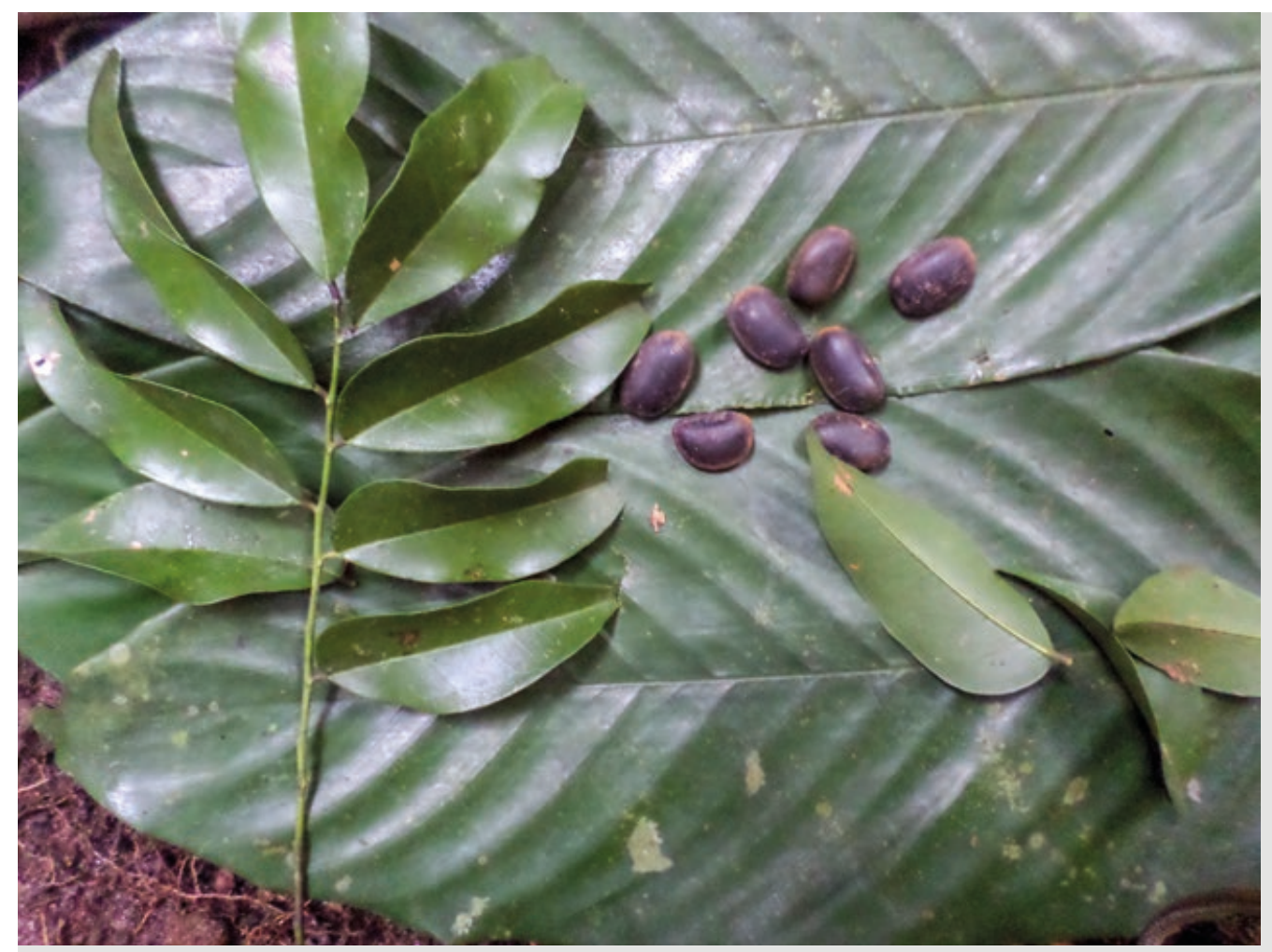

Photo 3.

Feuilles et graines de l'espèce Erythrophleum suaveolens (tali).

Photo C. Douh. 


\section{Influence des traitements sur la germination de la banque de graines du sol}

Les essais de Delhaye (2006) et Kouadio (2009) ont montré que les graines récoltées directement après dispersion et qui ne sont pas traitées (Trait.0 dans notre cas) présentaient des pourcentages de germination relativement faibles ( $0 \%$ et $32 \%$, respectivement) par rapport à celles traitées à l'acide sulfurique (70\% et $98 \%$, respectivement).

Des résultats similaires ont été obtenus pour Acacia auriculiformis Cunn. ex Benth., espèce dont les graines présentent une dormance tégumentaire comparable à celle d'E. suaveolens (Azad et al., 2011 ; Olatunji et al., 2012).

Dans notre étude, le traitement témoin (Trait.0) présente au contraire une probabilité de germination plus élevée $(19,14 \%)$ que les lots traités (4,34 \% et 15,21\%). Contrairement aux graines des études précédentes qui ont été récoltées directement sur le sol aux pieds des semenciers (Delhaye, 2006 ; Kouadio, 2009 ; Azad et al., 2011 ; Olatunji et al., 2012), la probabilité de germination plus élevée du traitement témoin (Trait.0) pourrait s'expliquer par le séjour des graines dans la banque du sol qui a probablement amorcé la levée de dormance, permettant à l'eau d'atteindre l'embryon de la graine.

La présente étude nous conduit à formuler l'hypothèse de la présence de deux types de graines dans le sol : des graines encore dormantes et des graines ayant achevé leur dormance. Les graines en fin de dormance auront la possibilité de germer lorsqu'elles seront mises dans des conditions favorables en termes d'hydratation, de température et d'oxygène (Augspurger et Kitajima, 1992 ; Dalling et al., 1998 ; Baraloto et al., 2005).

Pour les graines encore dormantes, un traitement à l'acide sulfurique $\left(\mathrm{H}_{2} \mathrm{SO}_{4}\right)$ devrait lever leur dormance. L'intensité de ce traitement devrait toutefois être adaptée en fonction du temps de séjour dans le sol, lequel ne peut être déterminé a priori.

\section{Longévité des graines dans la banque du sol}

Les graines d'E. suaveolens survivent au moins cinq à neuf ans dans le sol. Ce cas n'est pas unique. Des graines de Zanthoxylum ekmannii âgées de 15-16 ans ont été trouvées dans les trois premiers centimètres de la banque du sol; chez Trema micrantha, les âges varient entre 20 et 21 ans tandis que Croton billbergianus présente des semences pouvant rester viables dans la banque du sol pendant 38 ans (Dalling et Brown, 2009). Toutefois, le fait qu'aucune graine d'E. suaveolens n'ait été trouvée à plus de $20 \mathrm{~cm}$ de profondeur plaiderait en faveur d'une viabilité malgré tout limitée, laquelle confirmerait les résultats obtenus lors des traitements à l'acide.

\section{Implications sylvicoles}

L'ouverture du couvert forestier lors de l'exploitation forestière (création des parcs de chargement, routes, chablis) active la banque de graines du sol (Dupuy, 1998). Des graines autrefois enfouies dans la banque du sol se retrouvent en surface, ce qui expliquerait la régénération d'E. suaveolens en bordure de routes (Kouadio, 2009 ; Kleinschroth, 2016). Néanmoins, l'étendue de telles ouvertures du couvert forestier demeure limitée à $6 \%$ dans les forêts légalement exploitées (Kleinschroth, 2016), ce qui ne paraît pas suffisant pour pallier le déficit de régénération généralement observé pour cette espèce. Dans ce cas, des interventions sylvicoles basées sur des techniques de plantation ou d'enrichissement sont possibles pour contribuer à gérer de façon durable les forêts de production, afin d'assurer leur renouvellement et leur pérennité (Favrichon et al., 1997).

Ainsi, pour pallier les déficits locaux de régénération des plantules d'E. suaveolens, une régénération artificielle à partir de plantules produites en pépinière pourrait se faire en zone de forêt dégradée (Doucet et al., 2016). On veillera néanmoins à ne pas produire de plants à partir de graines récoltées sur des pieds isolés, du fait de la forte dépression de consanguinité observée chez cette espèce (Duminil et al., 2016).

\section{Conclusion}

Nos différentes hypothèses n'ont pu être confirmées. Nous avons effectivement montré que la densité de graines d'E. suaveolens était influencée par le type forestier, indépendamment de la densité de semenciers potentiels. La densité de graines diminue rapidement avec la profondeur et devient nulle à partir de $20 \mathrm{~cm}$.

Une levée progressive de la dormance et une plus forte vulnérabilité des graines aux pathogènes expliqueraient une survie se limitant à une dizaine d'années dans les sols tropicaux. L'exploitation forestière pourrait favoriser la germination d'une partie de ces graines. Mais cela ne permettra probablement pas de compenser les déficits de régénération observés pour cette espèce dans les forêts matures (Kouadio, 2009 ; Morin-Rivat et al., 2017). Si rien n'est fait pour compenser ce déficit, il est très probable que l'espèce régresse fortement au gré des rotations successives à l'instar d'autres espèces héliophiles comme l'ayous, Triplochiton scleroxylon (Karsenty et Gourlet-Fleury, 2006).

La plantation dans les milieux ouverts avec des plantules provenant des graines récoltées sur des pieds non isolés et conduites en pépinière apparaît nécessaire, voire essentielle.

\section{Remerciements}

Les auteurs remercient l'Université Marien Ngouabi (République du Congo), l'Université de Liège et sa faculté Gembloux Agro-Bio Tech (Belgique), le projet PEFOGRN-BC (Projet d'Appui au Programme Élargi de Formation en Gestion des Ressources Naturelles dans le Bassin du Congo). Le projet DynAfFor (Dynamique des forêts d'Afrique centrale) financé par le FFEM et l'AFD, l'asbl Nature+, ainsi que les entreprises forestières Mokabi-Dzanga (Groupe Rougier) et CIB/OLAM pour leurs soutiens technique, scientifique et financier. Le docteur Thierry Vanderborght du jardin botanique de Meise est également remercié pour le test final de coupe de graines. 
Bois et Forêts des Tropiques - ISSN: L-0006-579X

Volume $338-4^{\text {rd }}$ quarter - December 2018 - p. 43-55

52 FOCUS / SOIL SEED BANK OF ERYTHROPHLEUM SUAVEOLENS

\section{Annexe 1.}

Dispositif de collecte de la banque de graines du sol d'Erythrophleum suaveolens (les fosses, positionnées à $5 \mathrm{~m}$ de chaque semencier, mesurent $1 \mathrm{~m} \times 1 \mathrm{~m}$ ).

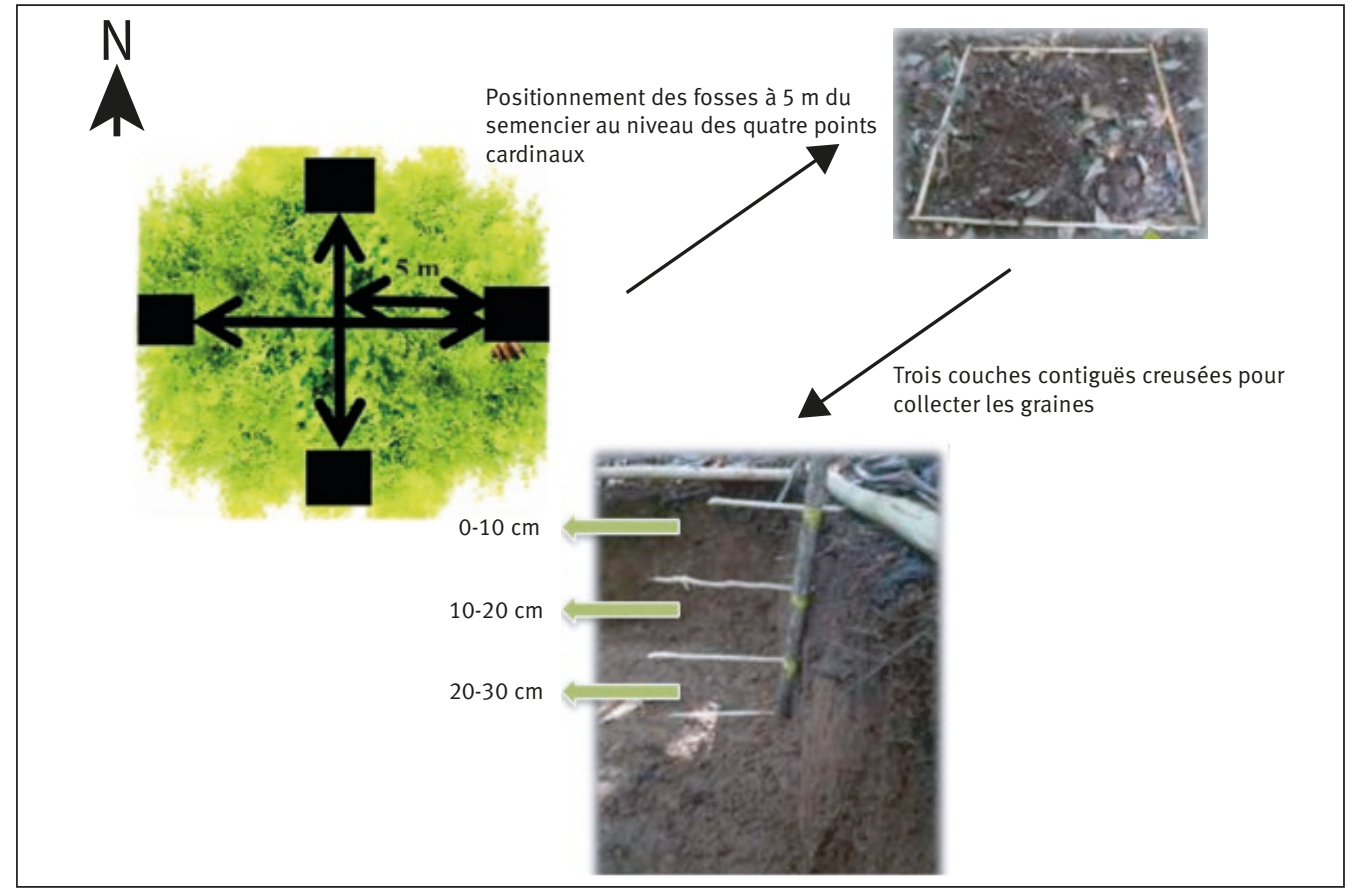

\section{Annexe 2.}

Probabilités associées aux cinq graines analysées. La datation radiocarbone effectuée sur les cinq graines indique que les graines datent soit de 1957 , mais avec une faible probabilité, soit de 2005-2009, avec une plus grande probabilité. Considérant l'année de collecte des graines, ici 2014, les graines seraient ainsi âgées d'au moins 5-9 ans.

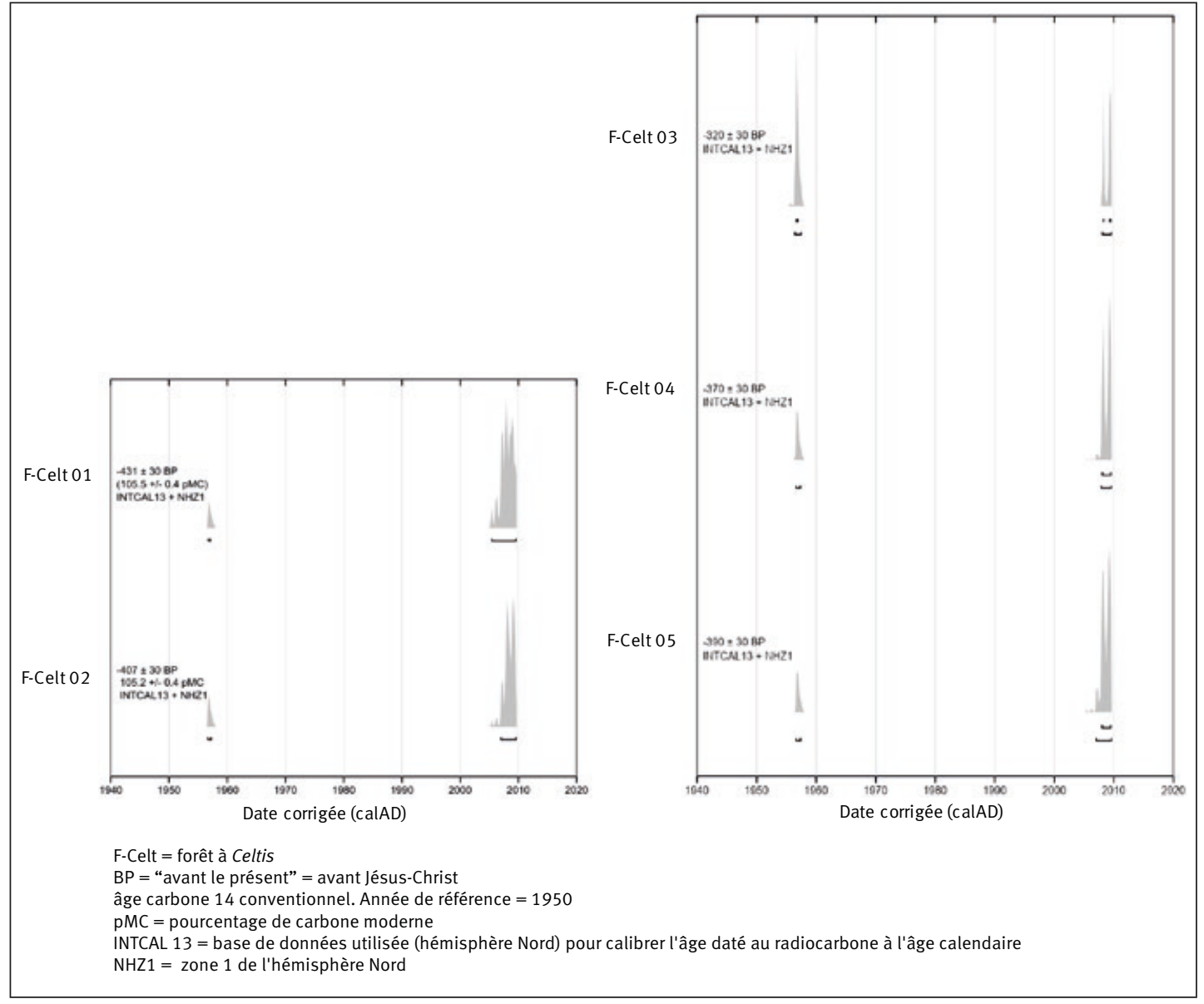




\section{Références bibliographiques}

Aubréville A., 1970. Flore du Cameroun. № 9. Légumineuses (Césalpinioidées). Paris, France, Muséum national d'histoire naturelle, $339 \mathrm{p}$.

Augspurger C. K., Kitajima K., 1992. Experimental studies of seedling recruitment from contrasting seed distributions. Ecology, 73: 1270-1284. https://doi.org/10.2307/1940675

Augspurger C. K., Kelly C. K., 1984. Pathogen mortality of tropical tree seedlings: experimental studies of the effects of dispersal distance, seedling density, and light conditions. Fcologia, 61: 211-217. https://doi.org/10.1007/BF00396763

Augspurger C. K., 1983. Seed dispersal of the tropical tree Platypodium elegans, and the escape of its seedlings from fungal pathogens. Journal of Ecology, 71: 759-771. https://doi. org/10.2307/2259591

Azad S., Manik M. R., Hasan S., Matin A., 2011. Effect of different pre-sowing treatments on seed germination percentage and growth performance of Acacia auriculiformis. Journal of Forestry Research, 22: 183-188. https://doi.org/10.1007/ s11676-011-0147

Baraloto C., Goldberg D. E., Bonal D., 2005. Performance tradeoffs among tropical tree seedlings in contrasting microhabitats. Ecology, 86: 2461-2472. https://doi.org/10.1890/04-1956

Baskin J. M., Baskin C., 2003. Classification, biogeography and phylogenetic relationships of seed dormancy. In: Smith R. D. et al. (eds). Seed conservation. Turning science into practice. Kew, UK, Kew Publishing, 517-544.

Bayol N., Anquetil F., Bile C., Bollen A., Bousquet M., Castadot B., Cerutti P., et al., 2014. The logging industry and management of natural forests: Tropical timber and the forests of Central Africa in the face of market trends. In: de Wasseige C., Flynn J., Louppe D., Hiol Hiol F., Mayaux Ph. (éds). The forests of the Congo Basin - State of the forest 2013. Neufchâteau, Belgique, Weyrich, 47-66.

Bronk Ramsey C., 1995. Radiocarbon calibration and analysis of stratigraphy: the OxCal program. Radiocarbon, 37 (2): 425-430. https://doi.org/10.1017/S0033822200030903

Cardoso F. C. G., Marques R., Botosso P. C., Marques M. C. M., 2012. Stem growth and phenology of two tropical trees in contrasting soil conditions. Plant Soil, 354: 269-281. https://doi. org/10.1007/s11104-011-1063-9

Chen H., Cao M., Tang Y., 2013. Soil seed banks in plantations and tropical seasonal rainforest of Xishuangbanna, South-West China. Journal of Tropical Forest Science, 25 (3): 375-386. https://search.proquest.com/ docview/1431089791? accountid=14630

Christensen M., 1989. A view of fungal ecology. Mycologia, 81: 1-19. https://doi.org/10.1080/00275514.1989.12025620

Crist T. O., Friese C. F., 1993. The impact of fungi on soil seeds: implications for plants and granivores in semiarid shrub-steppe. Ecology, 74: 2231-2239. https://doi.org/10.2307/1939576

Daïnou K., Bauduin A., Bourland N., Gillet J.-F., Fétéké F., Doucet J.-L., 2011. Soil seed bank characteristics in Cameroonian rainforests and implications for post-logging forest recovery. Ecological Engineering, 37: 1499-1506. https://doi.org/10.1016/i. ecoleng.2011.05.004

Dalling J. W., Brown T. A., 2009. Long-term persistence of pioneer species in tropical rain forest soil seed banks. American Naturalist, 173: 531-535. https://doi.org/10.1086/597221
Dalling J. W., Swaine M. D., Garwood, N. C., 1998. Dispersal patterns and seed bank dynamics of pioneer trees in moist tropical forest. Ecology, 79: 564-578. https://doi. org/10.1890/0012-9658

Danthu P., 1993. L'inhibition tégumentaire des graines de Faidherbia albida et d'Acacia raddiana, aspect anatomique et application pratique. Bois et Forêts des Tropiques, 238: 24-25. https://doi.org/10.19182/bft1993.238.a19762

Delhaye N., 2006. Mise en place d'une technique de régénération assistée en forêt dense humide gabonaise. Mémoire de fin d'études, Faculté universitaire des sciences agronomiques de Gembloux, Belgique, 99 p.

De Wasseige C., Flynn J., Louppe D., Hiol Hiol F., Mayaux Ph. (éds), 2014. Les forêts du Bassin du Congo : état des forêts 2013. Neufchâteau, Belgique, Weyrich, 325 p.

Diabate M., Munive A., De Faria S. M., Ba A., Dreyfus B., Galiana A., 2005. Occurrence of nodulation in unexplored leguminous trees native to the West African tropical rainforest and inoculation response of native species useful in reforestation. New Phytologist, 166: 231-239. https://doi. org/10.1111/i.1469-8137.2005.01318.x

Donahue D. J., 1995. Radiocarbon analysis by accelerator mass spectrometry. International Journal of Mass Spectrometry and Ion Processes, 143: 235-245. https://doi. org/10.1016/0168-1176(94)04132-Q

Doucet J.-L., 2003. L'alliance délicate de la gestion forestière et de la biodiversité dans les forêts du centre du Gabon. Thèse de doctorat, Faculté universitaire des sciences agronomiques de Gembloux, Belgique, 323 p.

Doucet J.-L., Daïnou K., Ligot G., Ouédraogo D. Y., Bourland N., Ward S. E., et al., 2016. Enrichment of Central African logged forests with high-value tree species: testing a new approach to regenerating degraded forests. International Journal of Biodiversity Science, Ecosystem Services \& Management, 12 (1-2). https://doi.org/10.1080/21513732.2016.1168868

Douh C., Daïnou K., Loumeto J. J., Moutsambote J.-M., Fayolle A., Tosso F., et al., 2018. Soil seed bank characteristics in two central African forest types and implications for forest restoration. Forest Ecology and Management, 409: 766-776. https://doi. org/10.1016/j.foreco.2017.12.012

Douh C., Daïnou K., Loumeto J. J., Fayolle A., Doucet J.-L., 2014. Explorer la banque de graines du sol pour mieux comprendre la dynamique de régénération des forêts tropicales africaines (synthèse bibliographique). Biotechnologie, Agronomie, Société et Environnement, 18: 558-565. http://hdl.handle. net/2268/174538

Duminil J., Daïnou K., Kombi Kaviriri D., Gillet P., Loo J., Doucet J.-L., Hardy O. H., 2016. Relationships between population density, fine-scale genetic structure, mating system and pollen dispersal in a timber tree from African rainforests. Heredity (Edinb), 116: 295-303. http://hdl.handle.net/2268/193386

Duminil J., Heuertz M., Doucet J.-L., Bourland N., Cruaud C., Gavory F., et al., 2010. CpDNA-based species identification and phylogeography: application to African tropical tree species. Molecular Ecology, 19: 5469-5483. https://doi. org/10.1111/j.1365-294X.2010.04917.x

Dupuy B., 1998. Bases pour une sylviculture en forêt dense tropicale humide africaine. Montpellier, France, Cirad, Document Forafri, 4, 328 p. http://catalogue-bibliotheques.cirad.fr/cgibin/koha/opac-detail.pl?biblionumber $=21340$ 
Durrieu de Madron L., Forni E., 1997. Aménagement forestier dans l'Est du Cameroun. Bois et Forêts des Tropiques, 254: 39-50. http://revues.cirad.fr/index.php/BFT/article/ view/19897

Effiom E. O., Nunez-Iturri G., Smith H. G., Ottosson U., Olsson O., 2013. Bushmeat hunting changes regeneration of African rainforests. Proceedings of the Royal Society of London B: Biological Sciences, 280 (1759): 2013.0246. http://dx.doi.org/10.1098/ rspb. 2013.0246

Elazazi E. M., 2016. Effective methods to improvement Capparis spinosa $\mathrm{L}$. (Caper) seeds germination by breaking seed dormancy in Qatar Gene Bank. Qatar Foundation Annual Research Conference Proceedings 2016: EEPP2483. http://dx.doi. org/10.5339/afarc.2016.EEPP2483.

Ensconet, 2009. Ensconet. Seed Collecting Manual for Wild Species. http://www.ensconet.eu/Download.htm

Evrard Q., Haurez B., Doucet J.-L., 2017. Le rôle des rongeurs dans la dispersion des diaspores en milieu forestier (synthèse bibliographique). Biotechnologie, Agronomie, Société et Environnement, 21 (1) : 66-79.

Favrichon V., Damio T., Doumbia F., Dupuy B., Higuchi N., Kokasi Kadir K., Maître H.-F., Nguyen-The N., Petrucci Y., Sist P., 1997. Réaction de peuplements forestiers tropicaux à des interventions sylvicoles. Bois et Forêts des Tropiques, 254 (4) : 5-24. http://revues.cirad.fr/index.php/BFT/article/view/19898

Fayolle A., Picard N., Doucet J.-L., Swaine M., Bayol N., Bénédet F., Gourlet-Fleury S., 2014. A new insight in the structure, composition and functioning of central African moist forests. Forest Ecology and Management, 329: 195-205. https://doi. org/10.1016/i.foreco.2014.06.014

Fayolle A., Engelbrecht B., Freycon V., Mortier F., Swaine M., Réjou-Méchain M., et al., 2012. Geological substrates shape tree species and trait distributions in African moist forests. PLoS One 7 (8): e42381. https://doi.org/10.1371/journal. pone.0042381

Footitt S., Cohn M. A., 1995. Seed dormancy in red rice (Oryza sativa). IX. Embryo fructose-2,6 bisphosphate during dormancy breaking and subsequent germination. Plant Physiology, 107: 1365-1370. https://doi.org/10.1104/pp.107.4.1365

Freycon V., 2014. Caractérisation des sols de Loundoungou et de Mokabi (Congo). Rapport de mission DynAfFor, 2-24 avril 2014. Montpellier, France, CIRAD. https://agritrop.cirad.fr/573670/1/ document 573670.pdf

Garwood N. C., 1989. Tropical soil seed banks: a review. In Leck M. A., Parker V. T., Simpson R. L. (eds). Ecology of soil seed banks. New York, USA, Academic Press, 149-209.

Gond V., Fayolle A., Pennec A., Cornu G., Mayaux P., Camberlain P., et al., 2013. Vegetation structure and greenness in Central Africa from Modis multi-temporal data. Philosophical Transactions of the Royal Society B: Biological Sciences, 368 (1625): 2012.0309. http://dx.doi.org/10.1098/rstb.2012.0309

Gorel A. P., Fayolle A., Doucet J.-L., 2015. Écologie et gestion des espèces multi-usages du genre Erythrophleum (Fabaceae-Caesapinioideae) en Afrique (synthèse bibliographique). Biotechnologie, Agronomie, Société et Environnement, 19 (4): 415-429.

Guion H., 2011. Étude de l'écologie d'Erythrophleum suaveolens (Guill. \& Perr.) Brenan (Tali) et de l'origine de ses populations au sud-est du Cameroun. Mémoire de fin d'études, Gembloux Agro-Bio Tech, Université de Liège, Belgique, 79 p.

Hall J. S., Swaine M. D., 1980. Seed stocks in Ghanaian forest soils. Biotropica, 12: 256-263. http://dx.doi. org/10.2307/23876959
Hardin J. W., Hilbe J. M., 2007. Generalized Linear Models and Extensions. $2^{\text {nd }}$ edition. College Station, USA, Stata Press.

Haurez B., Petre C.-A., Doucet J.-L., 2013. Impacts of logging and hunting on western lowland gorilla (Gorilla gorilla gorilla) populations and consequences for forest regeneration. A review. Biotechnologie, Agronomie, Société et Environnement, 17: 364372. http://hdl.handle.net/2268/152211

Hawthorne W. D., 1995. Ecological profiles of Ghanaian forest trees. Oxford, UK, Oxford Forestry Institute, Tropical Forestry Papers 29.

Hecketsweiler P., 1992. Phénologie et saisonnalité en forêt gabonaise. L'exemple de quelques espèces ligneuses. Thèse de doctorat, Université de Montpellier II, France, 266 p.

Hopkins M. S., Graham A. W., 1983. The species composition of soil seed banks beneath lowland tropical rainforests in North Queensland, Australia. Biotropica, 15: 90-99. http://dx.doi. org/10.2307/2387950

Hua Q., Barbetti M., Rakowski A. Z., 2013. Atmospheric radiocarbon for the period 1950-2010. Radiocarbon, 55: 2059-2072. https://doi.org/10.2458/azu js rc.v55i2.16177

Karsenty A., Gourlet-Fleury S., 2006. Assessing sustainability of logging practices in the Congo Basin's managed forests: the issue of commercial species recovery. Ecology and Society, 11: 26. http://www.ecologyandsociety.org/vol11/iss1/art26/.

Kleinschroth F., 2016. Roads in the rainforests: Legacy of selective logging in Central Africa. Doctoral dissertation, AgroParisTech, CIRAD, Bangor University. https://hal.archives-ouvertes. fr/tel-01366607/document.

Koné I., Lambert J., 2008. Primate seed dispersal and its potential role in maintaining useful tree species in the Taï region, Côte d'Ivoire: implications for the conservation of forest fragment. Tropical Conservation Science, 1 (3): 293-306. https://doi. org/10.1177/194008290800100309

Kouadio Y. L., 2009. Mesures sylvicoles en vue d'améliorer la gestion des populations d'essences forestières commerciales de l'Est du Cameroun. Thèse de doctorat, Faculté universitaire des sciences agronomiques de Gembloux, Belgique, 266 p.

Lawton R. O., Putz F. E., 1988. Natural disturbance and gapphase regeneration in a wind-exposed tropical cloud forest. Ecology, 69: 764-777. https://doi.org/10.2307/1941025

Lescure J.-P., De Foresta H., Riera B., 1989. Concepts communs à l'analyse des processus de dynamique et de succession. Bois et Forêts des Tropiques, 219: 57-60. http://revues.cirad.fr/index. $\mathrm{php/BFT/article/view/19646}$

Maguire J. D., 1962. Speed of germination-aid in selection and evaluation for seedling emergence and vigor. Crop Science, 2: 176-177.

Mapongmetsem P.-M., Duguma B., Nkongmeneck B. A., Puig H., 1998. Déterminisme de la défeuillaison chez quelques essences forestières tropicales du Cameroun. Revue d'Écologie, 53: 193-210. http://hdl.handle.net/2042/54895

Mbarga R. B., Jonkers W. B. J., Etoundi J., 1999. Phénologie de 86 essences productrices de bois d'œuvre de la forêt dense humide sempervirente du Sud-Cameroun - Résultats préliminaires. In: Nasi R., Amsallem I., Drouineau S. (éds). La gestion des forêts denses africaines aujourd'hui. Actes du séminaire Forafri de Libreville, Gabon. Montpellier, France, CIRAD-Forêt. http://www.tropenbos.nl/tbi publications/documents/FORAFRI-bibani.pdf 
Mbete P., Mopoundza P., Mbete R., Massang-Bienet., Ngokaka C., 2014. Impact du mode de prélèvement sur la faune de l'Unité Forestière d'Aménagement (UFA) Mokabi-Dzanga au nord du Congo Brazzaville. Journal of Applied Biosciences, 75: 6202-6210. http://dx.doi.org/10.4314/jab.v75i1.6

McCullagh P., Nelder J. A., 1989. Generalized Linear Models. 2nd edition. London, New York, Chapman \& Hall.

Mills J. T., 1983. Insect-fungus associations influencing seed deterioration. Phytopathology, 73: 330-335. http://dx.doi. org/10.1094/Phyto-73-330

Morin-Rivat J., Fayolle A., Favier C., Bremond L., Gourlet-Fleury S., Bayol N., et al., 2017. Present-day central African forest is a legacy of the 19th century human history. elife 6: e20343. http://dx.doi.org/10.7554/eLife.20343

Morin-Rivat J., Biwole A., Gorel A.-P., Vleminckx J., Gillet J.-F., Bourland N., et al., 2016. High spatial resolution of late-Holocene human activities in the moist forests of central Africa using soil charcoal and charred botanical remains. The Holocene, 26: 1954-1967. https://doi.org/10.1177/0959683616646184

Morin-Rivat J., Fayolle A., Gillet J.-F., Bourland N., GourletFleury S., Oslisly R., et al., 2014. New evidence of human activities during the Holocene in the lowland forests of the Northern Congo Basin. Radiocarbon, 56: 209-220. https://doi. org/10.2458/56.16485

Moriuchi K. S., Venable D. L., Pake C. E., Lange T., 2000. Direct measurement of the seed bank age structure of a Sonoran Desert annual plant. Ecology, 81: 1133-1138. https://doi. org/10.1890/0012-9658

OIBT, 2012. Examen annuel et évaluation de la situation mondiale des bois. Yokohama, Japon, Organisation internationale des bois tropicaux.

Okeyo J. M., 2006. Erythrophleum suaveolens (Guill. \& Perr.) Brenan. In: Schmelzer G. H., Gurib-Fakim A. (eds). PROTA (Plant Resources of Tropical Africa / Ressources végétales de l'Afrique tropicale). Wageningen, Netherlands. Consulté le $1^{\text {er }}$ mai 2018.

Olatunji D., Maku J. O., Odumefun O. P., 2012. Effect of pre-treatments on the germination and early seedlings growth of Acacia auriculiformis Cunn. ex. Benth. African Journal of Plant Science, 6: 364-369. https://doi.org/10.5897/AJPS11.255

Oslisly R., White L., Bentaleb I., Favier C., Fontugne M., Gillet J.-F., et al., 2013. Climatic and cultural changes in the west Congo Basin forests over the past 5000 years. Philosophical Transactions of the Royal Society of London B: Biological Sciences, 368 (1625): 2012.0304. http://dx.doi.org/10.1098/ rstb.2012.0304

Ouédraogo D. Y., Fayolle A., Gourlet-Fleury S., Mortier F., Freycon V., Fauvet N., et al., 2016. The determinants of tropical forest deciduousness: disentangling the effects of rainfall and geology in central Africa. Journal of Ecology, 104: 924-935. https://doi. org/10.1111/1365-2745.12589

Petre C.-A., Tagg N., Haurez B., Beudels-Jamar R., Huynen M.-C., Doucet J.-L., 2013. Role of the western lowland gorilla (Gorilla gorilla gorilla) in seed dispersal in tropical forests and implications of its decline. Biotechnology, Agronomy, Society and Environment, 17 (3): 517-526.

Picard N., Gourlet-Fleury S., 2008. Manuel de référence pour l'installation des dispositifs permanents en forêts de production dans le bassin du Congo. CIRAD, Rapport COMIFAC, 265 p.

Poorter L., Bongers F., Van Rompaey R., De Klerk M., 1996. Regeneration of canopy tree species at five sites in West African moist forest. Forest Ecology and Management, 84: 61-69. https://doi.org/10.1016/0378-1127(96)03736-X
Proctor J., Anderson J. M., Chai P., Vallack H. W., 1983. Ecological studies in four contrasting lowland rain forests in Gunung Mulu National Park, Sarawak. I. Forest environment, structure, and floristics. Journal of Ecology, 71: 237-260. https://doi. org/10.2307/2259975

Reimer P. J., Bard E., Bayliss A., Beck J. W., Blackwell P. G., Ramsey C. B., et al., 2013. IntCal13 and Marine13 radiocarbon age calibration curves 0-50,000 years cal. BP. Radiocarbon: 1869 1887. https://doi.org/10.2458/azu_js rc.55.16947

Saulei S. M., 1984. Natural regeneration following clear-fell logging operations in the Gogol Valley, Papua New Guinea. Ambio, 13: 351-354.

Sollins F., 1998. Factors influencing species composition in tropical lowland rain forest: does soil matter? Ecology, 79 (1): 23-30. https://doi.org/10.1890/0012-9658

Swaine M. D., Whitmore T. C., 1998. On the definition of ecological species groups in tropical rainforest. Vegetatio, 75: 81-86. https://doi.org/10.1007/BF00044629

Stokes E. J., Strindberg S., Bakabana P. C., Elkan P. W., Iyenguet F. C., Madzoké B., et al., 2010. Monitoring great ape and elephant abundance at large spatial scales: measuring effectiveness of a conservation landscape. PLoS One 5: e10294. https://doi. org/10.1371/journal.pone.0010294

Tosso F., Doucet J.-L., Migliore J., Daïnou K., Kaymak E., Kameni F. S. M., et al., 2017. Characterization of microsatellite markers in the African tropical tree species Guibourtia ehie (Fabaceae, Detarioideae). Applications in Plant Sciences, 5 (7): 1700023. https://doi.org/10.3732/apps.1700023

Uhl C., Clark K., 1983. Seed ecology of selected Amazon Basin successional species. Botanical Gazette, 144: 419-425. https:// doi.org/10.1086/337392

Uhl C., Clark K., Clark H., Murphy P., 1981. Early plant succession after cutting and burning in the upper Rio Negro region of the Amazon Basin. Journal of Ecology, 69: 631-649. https://doi. org/10.2307/2259689

Verelst L., 2009. Harmonized World Soil Database Viewer. FAO, CAS, IIASA, ISRIC, JRC. http://pure.iiasa.ac.at/8958

Warr S., Thompson K., Kent M., 1993. Seed banks as a neglected area of biogeographic research: a review of literature and sampling techniques. Progress in Physical Geography, 17: 329-347. https://doi.org/10.1177/030913339301700303

White F., 1983. The vegetation of Africa. A descriptive memoir to accompany the Unesco/AETFAT/UNSO Vegetation map of Africa. Paris, France, Unesco. https://doi.org/10.2307/633318

Bois et Forêts des Tropiques - Revue scientifique du Cirad
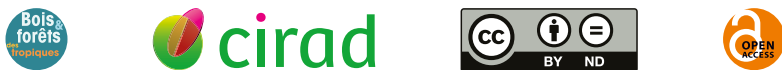

Cirad - Campus international de Baillarguet, 34398 Montpellier Cedex 5, France - Contact : bft@cirad.fr - ISSN : L-0006-579X 